\title{
Measuring Efficiency using Dynamic Network-Based Data Envelopment Analysis
}

\author{
Tsutomu Ito, Katsuhiko Takahashi, Katsumi Morikawa, Takao Ito \\ Graduate School of Engineering, Hiroshima University, 1-4-1 Kagamiyama, \\ Higashi-Hiroshima, 739-8527, Japan \\ E-mail:D152365@hiroshima-u.ac.jp \\ Rajiv Mehta \\ Martin Tuchman School of Management, New Jersey Institute of Technology, University Heights, \\ Newark, New Jersey, 07102-1982, U.S. \\ Makoto Sakamoto, Satoshi Ikeda \\ Faculty of Engineering, University of Miyazaki, 1-1 Gakuen Kibanadai-Nishi, \\ Miyazaki, 889-2192, Japan
}

\begin{abstract}
Enhancing efficiency is a critical objective of corporate management. While numerous approaches to measure efficiency have been devised, this manuscript suggests a new method referred to as dynamic network-based Data Envelopment Analysis (DEA) as a more advanced procedure for examining transaction priorities in any network based organization. Using dynamic network-based DEA it is possible to detect system-wide impacts when marginal systemic changes occur. This newer method was tested using data drawn from Mazda's Yokokai keiretsu to illustrate the effect that transactions among member firms in the network have on sales revenues. The finding of the study are discussed based on which managerial implications in the formulation of corporate strategy are proffered. Study drawbacks are identified and avenues for future research are suggested.
\end{abstract}

Keywords: DEA, dynamic network-based DEA, transaction priorities, Keiretsu, Yokokai.

\section{Introduction}

Ascertaining novel procedures for improving the efficiency of organizations is a continuing objective of corporate management. Although numerous tools to assess efficiency have been devised, this paper proposes a newer approach known as dynamic network-based Data Envelopment Analysis (DEA) as an even more effective approach for examining transaction priorities in any network. This is because using dynamic networkbased DEA enables the detection of systemic impacts when marginal changes in related inter-firm phenomena are manifest. Because of the scant research in this domain, this paper makes a unique contribution to the extant literature by: (1) Developing a new DEA model based on network analysis, and (2) Underscoring its function in corporate strategy.

This paper is organized as follows: Section 2 discusses the background and relevant literature focusing on DEA studies. In section 3, it offers a fivenode network to illustrate the problem using a standard DEA model, and details the process involved to resolve 
this issue. Section 4 explicates data collection procedures as well as calculates the efficiency of Yokokai-a typical network organization comprising suppliers of Mazda, a global auto manufacturer. The findings are reported and discussed following which the conclusions are presented in the final section.

\section{Background and Literature Review}

DEA refers to a linear programming method that assesses the efficiency of multiple decision-making units (DMUs) when the production process shows a structure of multiple inputs and outputs [1]. Originally proposed in 1957 by Farrell [2], it was popularized after Charnes, Cooper and Rhodes applied linear programming to estimate an empirical production technology frontier in 1978 [3]. Recently Liu and Lu developed network-based approach in applying one measure of the centrality - known as eigenvalue-to discriminate efficient decision-making organizations as determined by standard DEA modelling [4]. Chen indicated the importance of the intermediate outputs consumed by one sub DMU and suggested a networkDEA model with new efficiency operationalizations that systematically cope with the dynamic effect within a production network, such as a keiretsu [5].

Accordingly, this paper focuses on systemic changes in network systems to propose a new approach for calculating efficiency within keiretsu.

\section{Research Method}

A diagrammatic depiction among members of a small network of firms - as illustrated in Figure 1-reveals an asymmetric group of interconnected firms composed of five nodes. Nodes are called DMUs in standard DEA.

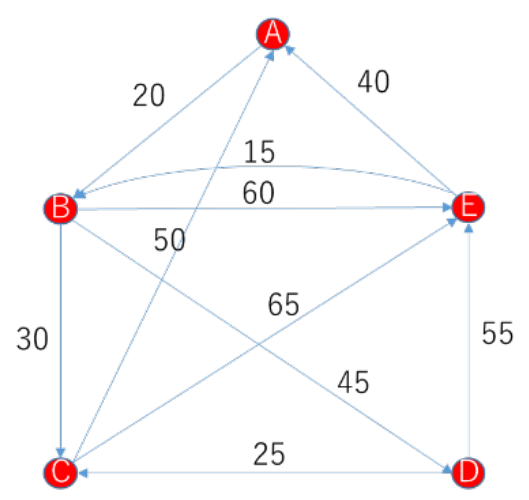

Figure 1. A five-node network.
For this type of an asymmetric network, it is possible to calculate the degree index, which can be dichotomized into out-degree and in-degree. The findings of outdegree and in-degree are $\{20,135,115,80,55\}$, and $\{90,35,55,45,180\}$ respectively. Suppose the sales of the five firms are 100,900, 250, 300, and 400, respectively. Based on the calculation of standard DEA model, the results are reported in Table 1.

Table 1. New results of Figure 1.

\begin{tabular}{|c|r|c|}
\hline & D Efficiency & Reference set $(\lambda)$ \\
\hline A & 0.688 & $5(0.250)$ \\
\hline B & 1 & $2(1.000)$ \\
\hline C & 0.412 & $2(0.251), 5(0.059)$ \\
\hline D & 0.557 & $2(0.297), 5(0.082)$ \\
\hline E & 1 & $5(1.000)$ \\
\hline
\end{tabular}

In Table 1, the efficiency of node A is 0.688 , which will improve if the out-degree and in-degree of the input data is changed to 0.6875 . After altering the input of out-degree and in-degree from 20 and 90 to 13.75 and 61.875 respectively, the newer results shows that the efficiency of node A has been improved. However, an important aspect, which is noteworthy, is that the other data remain constant even though out-degree and indegree of node A is changed. This result is seemingly inconsistent with the fact that systemic changes will be manifest even if a marginal adjustment has transpired. Thus, the input data should be recalculated. The new input of out-degree and in-degree are $\{13.5,135$, $99.375,80,42.5\}$, and $\{61.8759,28.75,55,45,180\}$, respectively. The results using the new input data are depicted in Table 2 .

Table 2. New results of Figure 1.

\begin{tabular}{|l|r|l|}
\hline & D Efficiency & \multicolumn{1}{|c|}{ Reference set $(\lambda)$} \\
\hline A & 0.773 & $\mathrm{E}(0.250)$ \\
\hline B & 1 & $\mathrm{~B}(1.000)$ \\
\hline C & 0.365 & $\mathrm{~B}(0.246), \mathrm{E}(0.072)$ \\
\hline $\mathrm{D}$ & 0.543 & $\mathrm{~B}(0.294), \mathrm{E}(0.089)$ \\
\hline $\mathrm{E}$ & 1 & $\mathrm{E}(1.000)$ \\
\hline
\end{tabular}

The efficiency of node A is 0.773 , which is smaller than 1 , which indicates that the DEA is not sufficiently suitable for detecting real systemic changes. Consequently, the DEA should be modified when it is used to measure efficiency. Thus, it is necessary to find an answer that makes the result of node $\mathrm{A}=1$. The best method is to employ a heuristic approach and calculate the input data at different levels. In this case, the efficient of node $\mathrm{A}$ will be 1 if the input data will be fixed at 45 percent $(0.45)$. The results of the input data analysis are reported in Figure 2. 


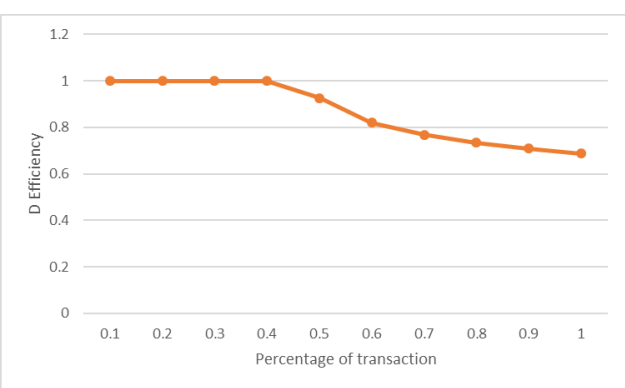

Figure 2. Heuristic results of the input data at different levels.

These findings are dependent on inputs that are difficult to adjust. Moreover, not all firms are necessarily completely efficient. Hence, setting the efficiency to one is an ideal objective. The DEA shows the reference set for improving efficiency, but does not provide any detailed directions for providing the optimal solution. Indeed, one of its limitations is that it treats the detailed information as a "black box." However, it is important to identify the importance of each company that it has ties with for the purposes of strategy formulation. Accordingly, this paper proposes a new index - known as the efficiency criterion - that is based on the simulation result of dynamic networkbased DEA. Therefore, the DEA is a useful tool not only to calculate the efficiency, but also to identify the relative importance of the relationship in any networkbased organization. Many methods, such as incremental approach and decremented approach, are considered useful.

Next, we test the new index known as the efficiency criterion to illustrate a detailed approach for Mazda's Yokokai using decremented approach. For purposes of illustration, we calculate the efficiency criterion using the data before discussing its implications for Mazda's Yokokai. Evidently, as shown in Figure 1, node A has three connections with $\mathrm{B}, \mathrm{C}$, and $\mathrm{E}$. The value between node $A$ and $B$ is 20 , which means node $A$ sells its 20 percent parts to node $\mathrm{B}$. The efficiency of all nodes is computed after removing a certain amount, for instance, fifty percent (see Table 3 ).

Table 3. Difference score of the trasanction of node A with B.

\begin{tabular}{|r|r|r|r|}
\hline & D Efficiency & $\begin{array}{c}\text { D Efficiency after } \\
\text { removing 50\% }\end{array}$ & $\begin{array}{c}\text { Difference } \\
\text { score }\end{array}$ \\
\hline $\mathrm{A}$ & 0.688 & 1 & 0.312 \\
\hline $\mathrm{B}$ & 1 & 1 & 0 \\
\hline $\mathrm{C}$ & 0.324 & 0.321 & -0.003 \\
\hline $\mathrm{D}$ & 0.557 & 0.551 & -0.006 \\
\hline $\mathrm{E}$ & 1 & 0.928 & -0.072 \\
\hline
\end{tabular}

As shown in Table 3, D efficiency is computed using original input data set and D efficiency after removing $50 \%$ (i.e., $50 \%$ of 20 ) is recalculated with the input data set employing standard DEA modelling. The difference score is defined as the gap between D Efficiency and D Efficiency after removing a certain amount, say $50 \%-$ as it is in this example. The efficiency has improved if the value is positive. The difference score of node A is 0.312 , which is suggestive that the efficiency will change if the transaction between node $\mathrm{A}$ and $\mathrm{B}$ decreases by $50 \%$. The difference score of node $B$ is zero. Evidently, there is no impact on node $\mathrm{B}$ if the amount between node $\mathrm{A}$ and $\mathrm{B}$ will be reduced to 10. Conversely, the efficiency will be low if the amount between node $\mathrm{A}$ and $\mathrm{E}$ decreases. Therefore, node $\mathrm{A}$ will have benefits if node $\mathrm{A}$ changes the transaction amount with node B.

The groups can be trichotomized in this transaction network. The first group is the selected nodes, the second group refers to the nodes dealing with the selected node, and the third group comprises the remainder of the nodal firms. However, it excludes the selected node and the respective nodes dealing with the selected nodes. Next, it is necessary to calculate the difference score of these three groups and determine the priority of each node based on the results of the selected node. The difference score of the three groups is shown in Figure 3.

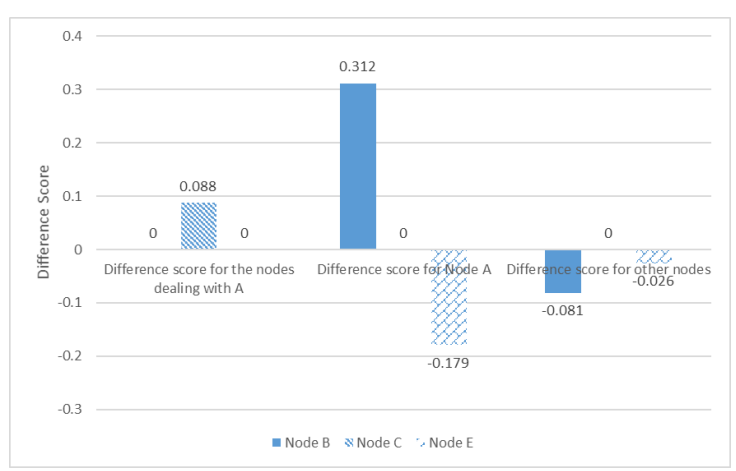

Figure 3. Difference score of node A with B, C, and E.

The summation of the difference score-called the difference criterion - will be considered as an index that identifies the transaction priority in any network. The difference criterion refers to the summation of the gap of all nodes' efficiency between the efficiency of the new system after changing a certain amount and the original system when the solution is fixed at one 
selected node. Essentially, this indicates the impact of each node. The difference criterion of node A is shown as in Figure 4.

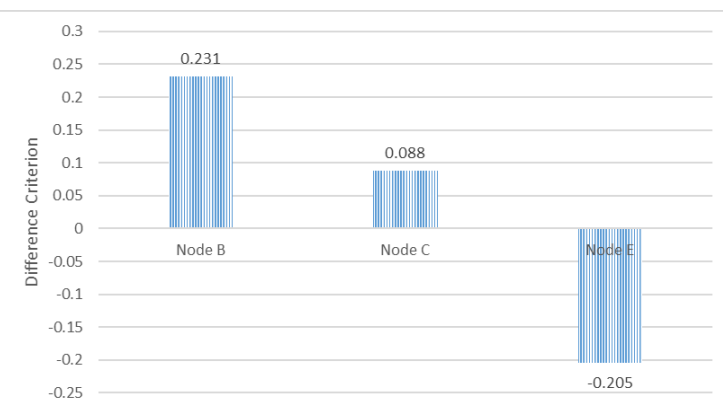

Figure 4 Difference criterion of node A with B, C, and E.

Accordingly, the priority of node A improving its efficiency is to focus on nodes B, and C. Node E should be excluded because its value is negative. As such, the efficiency criterion can be considered as an index of the impact of the selected company. This reveals that as the efficiency criterion gets larger, its impact gets stronger.

The step-wise procedure of the dynamic networkbased DEA is described next.

1) Building problem formulation: Many types of the DEA have been developed; the most typical one is known as the Charnes-Cooper-Rhodes (CCR) model. The generalized model of the CCR model is formulated as follows:

$\max \quad \theta=\frac{\sum_{r=1}^{s} u_{r} y_{r^{j}}}{\sum_{i=1}^{m} v_{i} x_{i j}}=\frac{u_{1} y_{1 j}+u_{2} y_{2 j}+\cdots+u_{s} y_{s j}}{v_{1} x_{1 j}+v_{2} x_{2 j}+\cdots+v_{m} x_{m j}}$

Subject to

$$
\begin{aligned}
& \frac{\sum_{r=1}^{s} u_{r} y_{r j}}{\sum_{i=1}^{m} v_{i} x_{i j}} \leq 1 \quad(j=1,2, \ldots, n) \\
& u_{r} \geq 0 \quad(r=1,2, \ldots, s) \\
& v_{i} \geq 0 \quad(i=1,2, \ldots, m)
\end{aligned}
$$

2) Calculating Efficiencies: The efficiencies are calculated using input and output data. The solution, or reference set, for a selected node with lower efficiency can, thus, be found.

3) Recalculating Data Set: The input and/or output data should be recalculated based on the reference set using the network model.

4) Recalculating Efficiencies: The efficiencies should be recalculated using the new data set calculated in third step. New results will be ascertained.
5) Finding new results: The calculation will be completed if the answer of the selected node is 1 ; otherwise, the answer should be ascertained by employing heuristic approach.

6) Determining the priorities: Finally, it is necessary to calculate the difference scores and difference criterion of three groups to determine the priority of each node deal with the selected node.

\section{Measurements and Discussion}

To study inter-firm relationships among keiretsu members, transaction data were obtained from Mazda's Yokokai, which represents a typical automotive manufacturer and parts-supplier keiretsu to calculate the efficiency of each company. It is noteworthy that the number of the firms dealing with Mazda usually fluctuates year to year. The total membership of the Yokokai in 2004 was 188, but the total firms Mazda dealt with was 91 .

The linkage between two firms is expressed as degree. Degree, including out-degree and in-degree, are calculated. Out-degree means that one company sells parts to other company, and in-degree means that one company purchases the parts from that same supplier firm. Degree is considered as the input data, and sales revenues are considered as the output data.

Mazda's Yokokai is divided into two groups comprising suppliers and automotive manufacturers. In the auto manufacturer group, the top three companies are No. 39, Pioneer Corporation, No.76, Matsushita Electric Industrial Co., Ltd., and No.45, Bosch Corporation. The efficiency of Pioneer Corporation and Matsushita Electric Industrial Co., Ltd. is one. Moreover, the efficiency of Bosch Corporation is 0.954 . Conversely, the efficiency of No.55 of Aisin AI Co., Ltd. and No. 21 of Jatco Ltd., are the lowest as they are 0.002 and 0.003 , respectively. They are subsidiaries of Toyota, and the distance to Mazda is considered as long than other suppliers. In the group of automakers, the highest value of efficiency is Toyota (0.01), and the lowest is Mazda (0.001).

To ascertain the detailed approach for improving a firm's efficiency, the first step is to compute the efficiency of all firms in Yokokai. To determine the best solution for suppliers, Unipres is selected for illustrative purposes. The primary business of Unipres, one of several Yokokai members, is to manufacture automotive and electronic parts that are sold to not only Mazda, but 
also non-keiretsu members, such as Nissan, Isuzu, Toyota and Jatco. Unipres also purchases parts from Yorozu Corporation, which also manufactures automobile suspension systems. Moreover, Unipres and Yorozu are members of an independent group of autopart suppliers.

The efficiency of Unipres is 0.0261. From these results, it can be determined that the objective of Unipres is to improve its efficiency, which can be accomplished by changing its input data for out-degree and in-degree 0.0261 times. Thus, this results in an efficiency of Unipres of 0.996 when the input data is changed 0.0261 times.

Based upon efficiency results, and the procedure of dynamic network-based DEA, D efficiency is calculated. The value is 0.0258 if $\mathrm{D}$ efficiency is set as 1 .

Next, to find the optimal solution of Unipres, the detailed transaction information in Yokokai should be analyzed, which is reported in Table 4. Unipres purchases the parts from Yorozu and sells its products to Mazda, Calsonic Kansai, Jatco, Nissan and Isuzu. Difference score of all firm inter-relationships with Unipres are depicted in Figure 5.

Table 4. Transaction of Unipres in Yokokai.

\begin{tabular}{|l|r|r|}
\hline & \multicolumn{1}{|c|}{ To Unipres } & \multicolumn{1}{c|}{ From Unipres } \\
\hline Mazda & 0 & 1.1 \\
\hline Calsonic Kansei & 0 & 3.5 \\
\hline Jatco & 0 & 10.8 \\
\hline Nissan & 0 & 58.9 \\
\hline Isuzu & 0 & 0.7 \\
\hline Yorozu & 1.6 & 0 \\
\hline
\end{tabular}

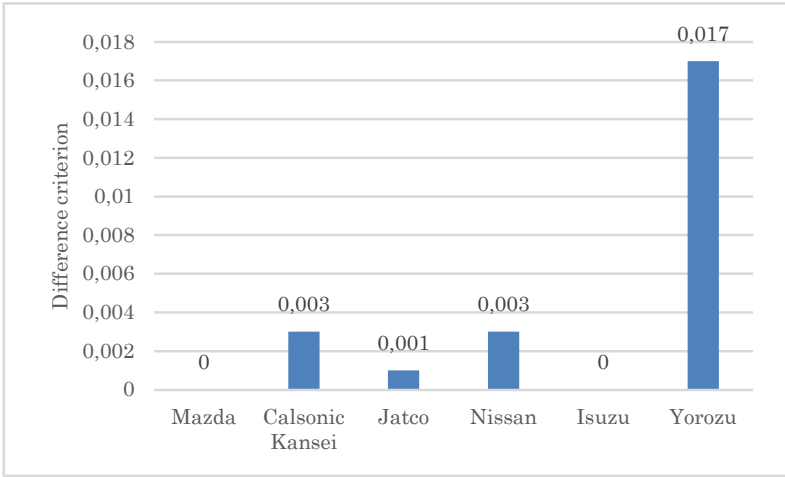

Figure 5. Difference score of Unipres.

The Difference criterion between Unipres and Yorozu is 0.017, and the value between Unipres and Nissan and Calsonic Kansai is 0.003 respectively. Therefore, Unipres should prioritize its business with Yorozu, Nissan, and Jatco.
Two additional examples are Japan Climate Systems Corporation and the renowned Sumino, which are members of Yokokai. Climate Systems is a firm that manufactures air-conditioning, condensers, and engine cooling systems. In 2004, Climate Systems sold its parts to Mazda while it purchased from Sumino, a renowned manufacturer of precision press welding components for automobiles in Yokokai. The detailed transaction information of Climate Systems is shown in Table 5 and 6.

Table 5. Transaction of Climate Systems in Yokokai.

\begin{tabular}{|l|l|l|}
\hline & \multicolumn{1}{|c|}{$\begin{array}{c}\text { To Climate } \\
\text { Systems }\end{array}$} & \multicolumn{2}{|c|}{$\begin{array}{c}\text { From Climate } \\
\text { Systems }\end{array}$} \\
\hline Mazda & 0 & 92 \\
\hline Sumino & 2 & 0 \\
\hline
\end{tabular}

Table 6. Transaction of Sumino in Yokokai.

\begin{tabular}{|l|r|r|}
\hline & \multicolumn{1}{|c|}{ To Sumino } & From Sumino \\
\hline Climate Systems & 0 & 2 \\
\hline NSK & 0 & 2 \\
\hline Mazda & 0 & 83 \\
\hline
\end{tabular}

Difference criterion of all firms associated with Climate Systems and Sumino are illustrated in Figures 6 and 7.

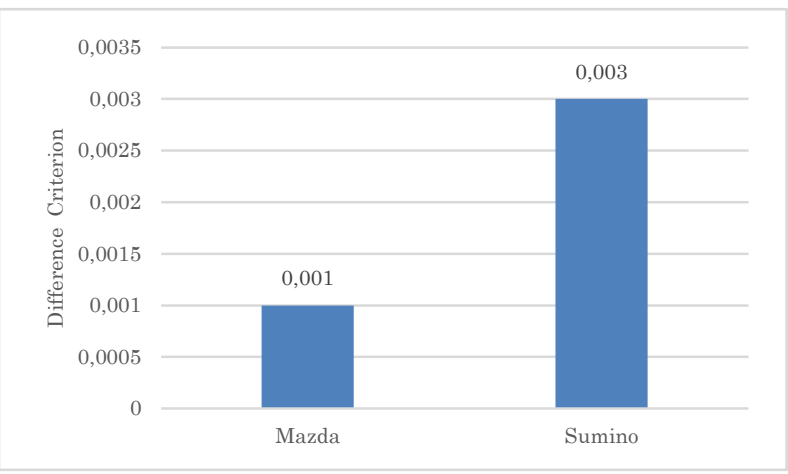

Figure 6. Difference criterion of Climate Systems.

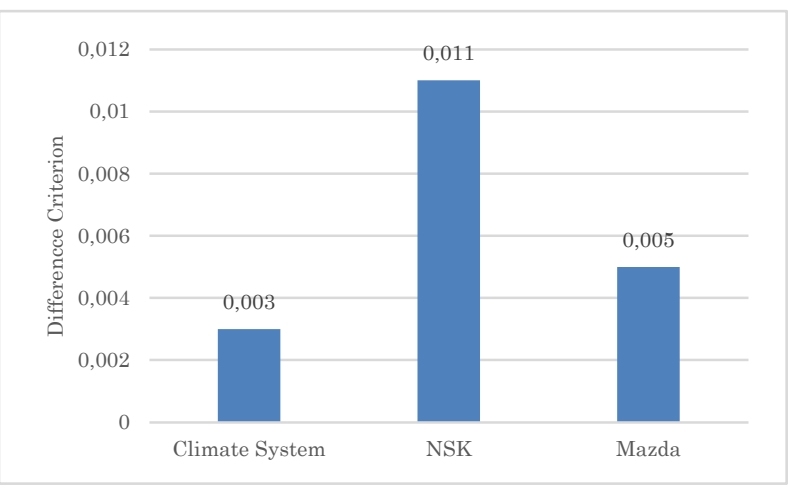

Figure 7. Difference criterion of Sumino. 
Figure 6 indicates that Climate Systems should prioritize the number of transactions with Sumino and Mazda. Figure 7 shows that the priority of transactions of Sumino is NSK, Mazda, and Climate Systems. Therefore, not only Climate Systems and Sumino, but also Mazda has the opportunity to improve their efficiency if the data between Climate Systems and Sumino is altered. Moreover, it is a mutual but asymmetric relationship between Climate Systems and Sumino, The priority of Climate Systems is Sumino and Mazda, but the priority of Sumino is NSK, Mazda, and Climate Systems. Climate Systems will get much more benefits to adjust its transaction amount compared with that of Sumino.

The results of this study makes several contributions to the extant literature to yield some implications that offer guidelines for managerial actions. More specifically, to augment corporate efficiency, the following suggestions for managing the interrelationships among keiretsu members are identified: First, this study proposed a new approach to identify systemic changes in a given network system. It clarified the internal solutions to link with the DEA model and network approach. In this paper, the efficiency of all firms was measured as well as the priority of the different firms was determined. Second, new indexes, such as differece sores and difference criterion were proposed. These new indexes express the changes after the data are modified. Third, calculating the efficiency in a given network is a useful tool because the relationship between a member vis-à-vis the whole network system can be developed.

\section{Conclusion}

In this paper, dynamic network-based DEA was proposed as a new approach for analyzing interrelationships among members of a keiretsu. Using transaction data drawn from Mazda's Yokokai, the efficiency of all member firms were calculated. The paper also suggested a new index-the efficiency criterion - to determine the importance of each firm in a given network.

A key limitation of this paper, however, is that only one network index-degree-is used. Additional network indices, such as betweenness, and density, should be investigated. Furthermore, in future research investigations not only transactional data, but also crossshareholdings among member firms, should be applied, which will enhance our understanding of how keiretsus can be strategically managed to yield higher performance objectives.

\section{Acknowledgements}

This work was supported by JSPS KAKENHI Grant Number 16K01243.

\section{References}

1. Yishi Zhang, Anrong Yang, Chan Xiong, Teng Wang, Zigang Zhang (2014). Feature selection using data envelopment analysis, Knowledge-Based Systems, Vol. 64, pp. 70-80, July 2014, ELSEVIER.

2. Farrell M. J., The Measurement of Productive Efficiency, Journal of the Royal Statistical Society, Series A (General) Vol. 120, No. 3, pp. 253-290, 1957, Royal Statistical Society (Wiley).

3. Charnes A., Cooper W.W., Rhodes E., Measuring the efficiency of decision-making units, European Journal of Operational Research, 2, pp.429-444, 1978, North-Holland Publishing Company.

4. J. S. Liu, W. Lu, DEA and ranking with the network-based approach: a case of $R \& D$ performance, Omega 38, pp.453-464, 2010, Elsevier.

5. Chien-Ming Chen, A network-DEA model with new efficiency measures to incorporate the dynamic effect in production networks, European Journal of Operational Research 194, pp.687-699, 2009, Elsevier. 